\title{
Lifetimes and electromagnetic transition strength in ${ }^{124} \mathrm{Ba}$
}

\author{
Mariya Trichkova ${ }^{1, *}$, Marcel Beckers ${ }^{2}$, Kalin Gladnishki1 ${ }^{1, * *}$, Alfred Dewald ${ }^{2}$, Andrey Blazhev ${ }^{2}$, Claus Müller- \\ Gatermann $^{2}$, Christoph Fransen ${ }^{2}$, Jan Jolie ${ }^{2}$, Desislava Kalaydjieva ${ }^{1}$, Georgi Rainovski ${ }^{1}$, and Karl-Oskar Zell ${ }^{2}$ \\ ${ }^{1}$ Faculty of Physics, St. Kliment Ohridski University of Sofia, Sofia, Bulgaria \\ ${ }^{2}$ Institut für Kernphysik, Universität zu Köln, Cologne, Germany
}

\begin{abstract}
Lifetimes of excited states of ${ }^{124} \mathrm{Ba}$ were measured by the Recoil Distance Doppler Shift (RDDS) technique. The $\gamma$-ray coincidence data were analysed by the Differential Decay Curve method (DDCM). The trend of the experimentally deduced normalized $B(E 2)$ values reveals a drop at the $8_{1}^{+}$state, which may be caused by structural changes in the backbending region.
\end{abstract}

\section{Introduction}

Barium isotopes are subject of considerable interest, because of a variety of structural phenomena observed in them. Backbends have been reported in ${ }^{126} \mathrm{Ba}[1],{ }^{128} \mathrm{Ba}$ [2] and ${ }^{130} \mathrm{Ba}$ [3] at the spin of $J^{\pi}=10^{+}$. In some cases, two "superbands" $[1,3]$ have been observed, and interpreted to arise from the particle aligned states involving protons or neutrons in the $h_{11 / 2}$ orbital. In addition to high-spin phenomena, $\gamma$-bands have been also observed. Their notable feature is the strong staggering of the excitation energies.

The light Barium isotope ${ }^{124} \mathrm{Ba}$ belongs to the transitional region between spherical and axially deformed nuclei. The level scheme of this nucleus was established through several spectroscopic measurements using heavyion reactions [4-8]. Several bands were established up to very high spin states. At spin $10^{+}$the ground state band of ${ }^{124} \mathrm{Ba}$ splits into two bands due to alignment of a pair of protons and neutrons in the $h_{11 / 2}$ orbitals at rotational frequencies 0.37 and $0.41 \mathrm{MeV}$, respectively. Possible configurations of these bands were discussed earlier using the cranked shell model $[5,7,8]$. It was suggested that the proton and neutron aligned bands are built on the $[550] 1 / 2^{-}$and $[523] 7 / 2^{-}$Nilsson orbitals, respectively. The proton band shows a second crossing at rotational frequency $0.49 \mathrm{MeV}$ with an alignment gain of $3.2 \hbar$ due to the alignment of the neutron $\mathrm{h}_{11 / 2}$ orbitals. Thus, the configuration changes from the zero quasiparticle ground-state band (g.s.b.) to a two-quasiparticle configuration [g.s.b. $-\pi\left(h_{11 / 2}\right)^{2}$ ] and then to a four quasiparticle structure [g.s.b. $-\pi\left(h_{11 / 2}\right)^{2}-v\left(h_{11 / 2}\right)^{2}$ ] above the rotational frequency $\hbar \omega=0.49 \mathrm{MeV}$.

The present work is focused on the low-lying levels in the yrast band of ${ }^{124} \mathrm{Ba}$. Their energies suggest the presence of X(5) [9] features in the investigated nucleus (see Fig. 1). The $X(5)$ model provides parameter free prediction for the level scheme and $B(E 2)$ transition strengths

\footnotetext{
*e-mail: mariya.trichkova@gmail.com

**e-mail: kag@phys.uni-sofia.bg
}

for nuclei at the critical point of the transition from spherical to axially deformed shape. The decisive experimental signatures for $\mathrm{X}(5)$ behavior is the evolution of $B(E 2)$ transition strengths with increasing the spin [9]. Therefore we have performed Recoil Distance Doppler Shift (RDDS) measurements in order to determine lifetimes of the yrast band states in ${ }^{124} \mathrm{Ba}$ isotope, from which to calculate the respective $B(E 2)$ values.

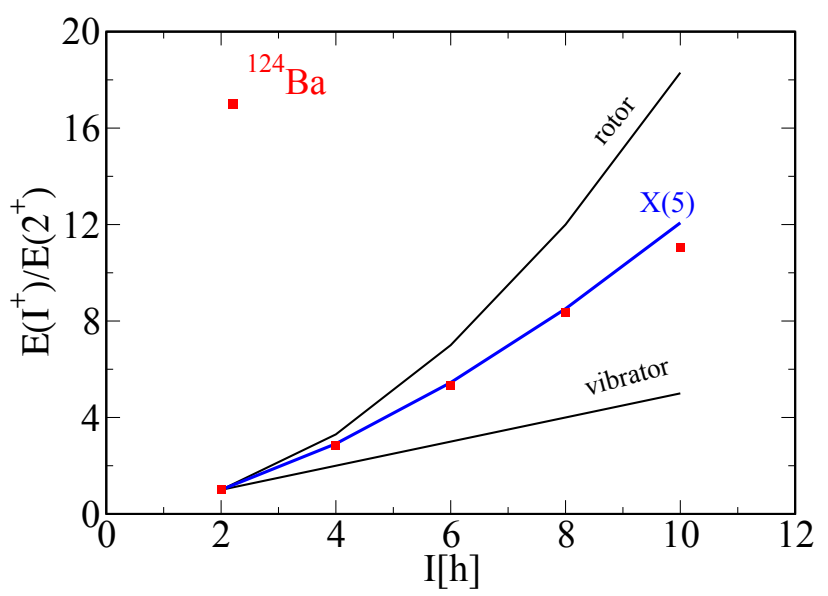

Figure 1. Comparison between experimentally observed energies of the levels in g.s.b. and those predicted from the vibrator, rotor and $\mathrm{X}(5)$ theoretical approaches.

\section{Experimental details}

Excited states in ${ }^{124} \mathrm{Ba}$ were populated via the ${ }^{105} \mathrm{Pd}\left({ }^{23} \mathrm{Na}, 1 p 3 n\right)$ reaction. The beam with an energy of $93 \mathrm{MeV}$ was provided by the FN Tandem accelerator of the Institut für Kernphysik at the University of Cologne. The target consisted of self supporting $0.65 \mathrm{mg} / \mathrm{cm}^{2}$ 
Ring $2\left(142^{\circ}\right)$

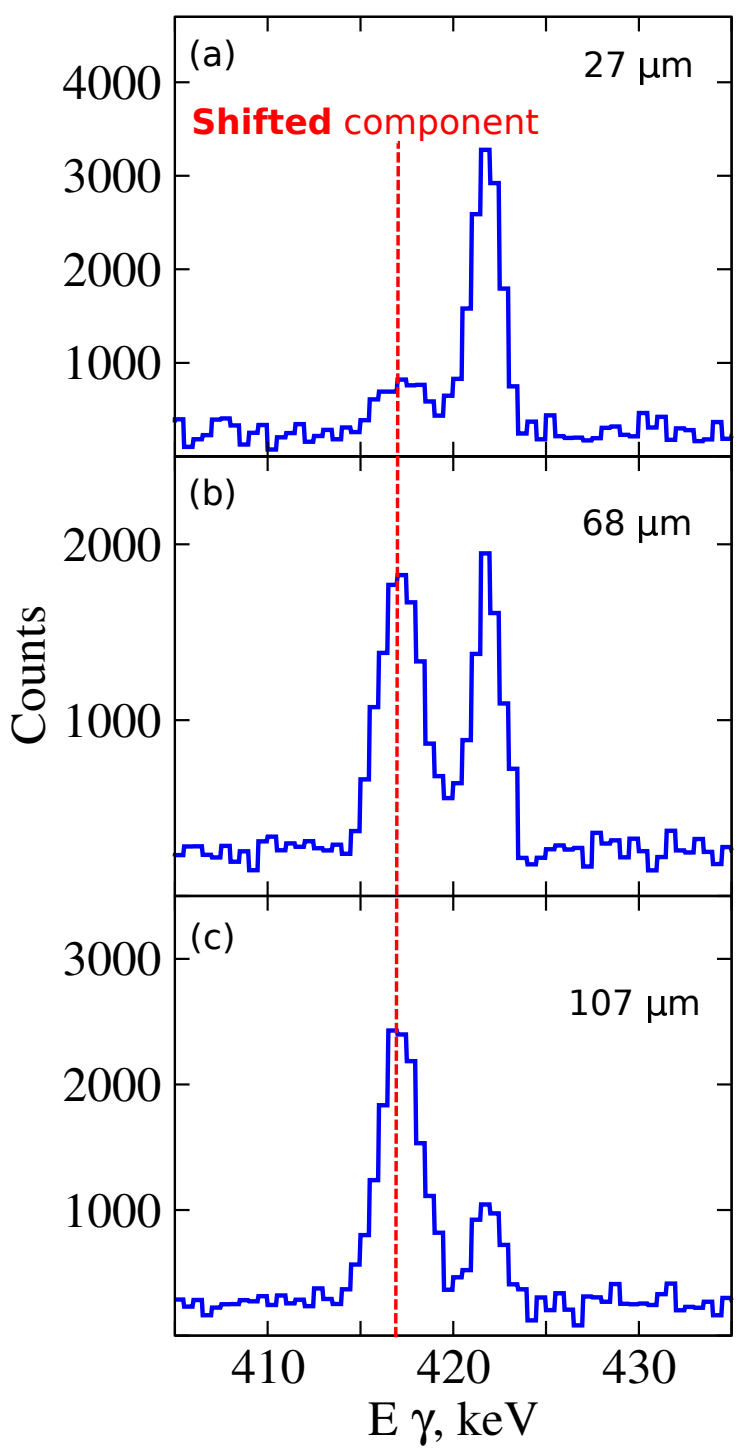

Ring $1\left(45^{\circ}\right)$

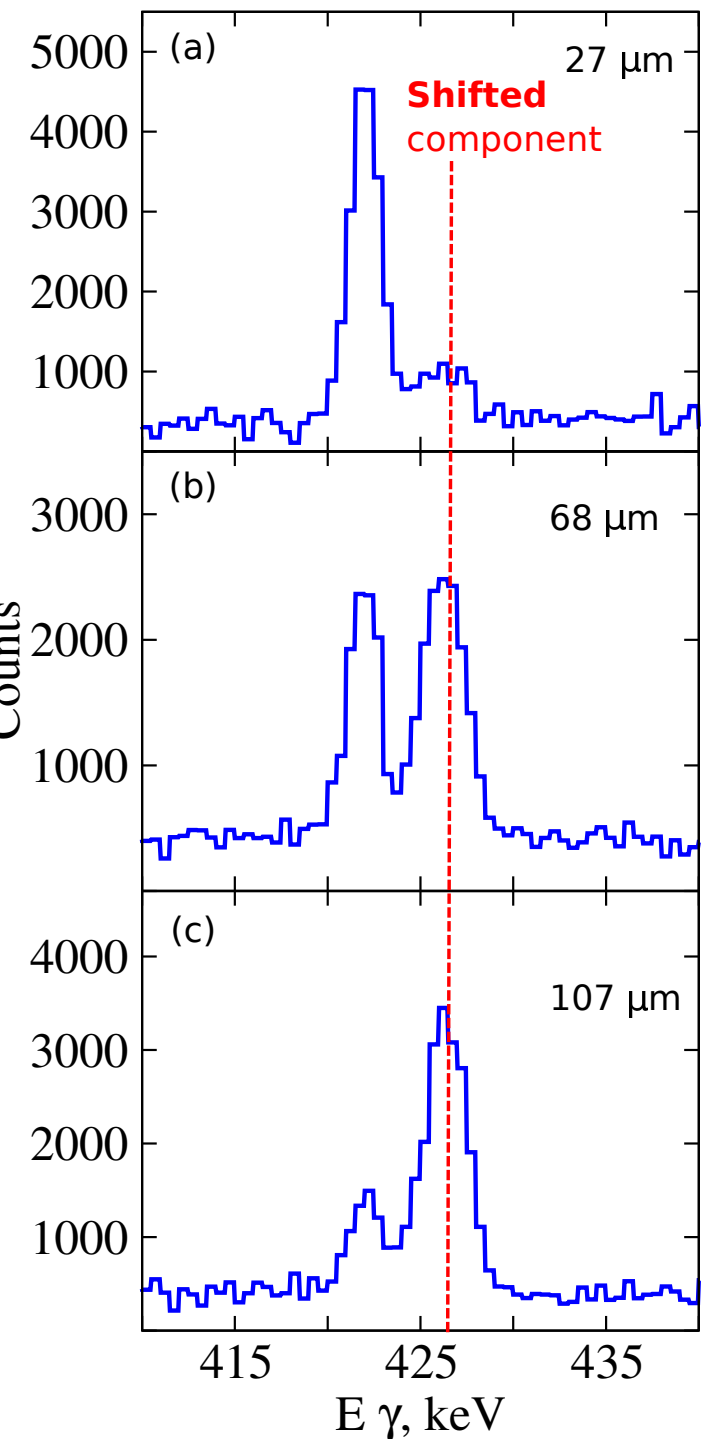

Figure 2. (Color online) The Doppler-shifted and unshifted peaks of the $421.1 \mathrm{keV} 4_{1}^{+} \rightarrow 2_{1}^{+}$transition, observed in backward and forward detectors at multiple distances. The gate is applied on the shifted component of the $6_{1}^{+} \rightarrow 4_{1}^{+}$transition.

thick Pd foil (enriched up to $94 \%$ with the isotope ${ }^{105} \mathrm{Pd}$ ) followed by a Ta stopper foil of $7.3 \mathrm{mg} / \mathrm{cm}^{2}$ thickness.

The target and stopper foils were mounted in the Cologne plunger device [10]. The constancy of the selected target-to-stopper distance is controlled by measuring the target-stopper capacity and by compensating drifts with a piezo-electric device. The different distances used during the measurement are set by moving the target holder. Deexciting $\gamma$-rays were registered with a setup consisting of 12 large-volume High Purity Germanium detectors (HPGe) mounted outside the plunger chamber. The detectors were grouped in three rings, namely ring 0 ( 1 detector on polar angle of $0^{\circ}$ with respect to the beam axis), ring 1 (six detectors on polar angle of $45^{\circ}$ ) and ring 2 (five detectors on polar angle of $142^{\circ}$ ).

\section{Data analysis and preliminary results}

The Recoil Distance Doppler Shift (RDDS) method is a well known technique for the determination of picosecond lifetimes of excited nuclear states (for more details see e.g. Ref. [11] and references therein). Using this method we measured lifetimes of yrast states in ${ }^{124} \mathrm{Ba}$. For each level of interest the properly normalized intensities of the shifted $\left(I_{s}\right)$ and unshifted $\left(I_{u}\right)$ components of the deexciting transition were determined from coincidence spectra with the shifted component of a transition directly populating the state for each distance. In this way problems with unobserved sidefeeding are eliminated. In addition, by gating only on the shifted component of a feeding transition the effect of nuclear deorientation does not influence the results of the lifetime analysis [12]. Normalization was done to account for the different number of the observed 


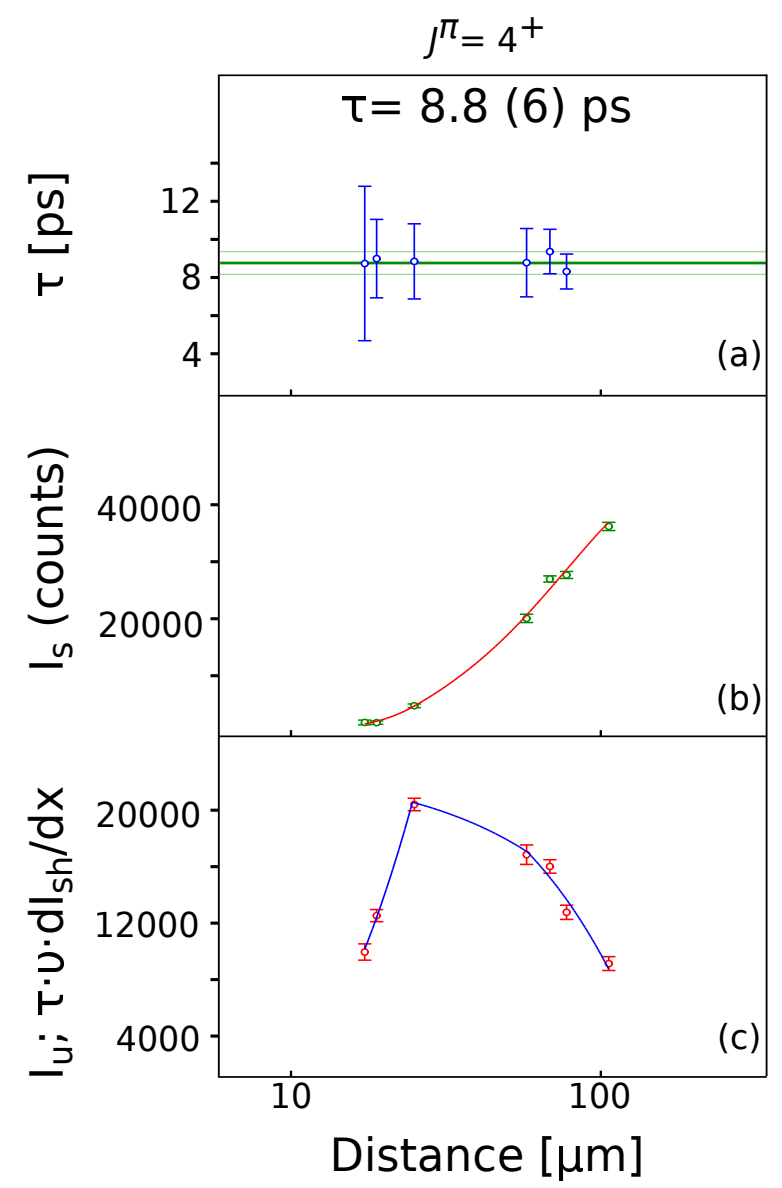

Figure 3. (Color online) DDCM analysis for the $4_{1}^{+}$state after gating on the backward detectors and projecting the backward spectra. (a) Lifetimes determined for each distance setting in the sensitive region, the horizontal line represents the mean value. (b) Normalized intensity curve for the shifted component, $I_{s}$ of the transition depopulating the $4_{1}^{+}$state and fitted spline curve. (c) Normalized intensity curve for the unshifted component, $I_{u}$. The curve in (c) is proportional to the derivative of that in (b) and both curves are fitted simultaneously. According to Eq. (1), the ratio of these gives the lifetime of the state, shown in (a). The distance between the target and the stopper is given on the abscissa.

decays for each distance. The lifetimes were extract employing the Differential Decay Curve method (DDCM), presented in $[13,14]$.

According to the DDCM, if the level of interest is populated by transition $\mathrm{B}$ and it is depopulated via transition $\mathrm{A}$, in the special case of gating on the Doppler-shifted component of the direct feeding transition (B), the mean lifetime $\tau$ can be derived for each target-to-stopper distance by applying the following equation:

$$
\tau_{i}(x)=\frac{I_{u}^{B A}(x)}{v \cdot \frac{d}{d x} I_{s}^{B A}(x)},
$$

where $v$ denotes the recoil velocity. The quantities $I_{u}^{B A}(x)$ and $I_{s}^{B A}(x)$ denote the normalized, measured intensities of the unshifted (u) and shifted (s) components of the depopulating $\gamma$ transition A in coincidence with the shifted component of a populating $\gamma$ transition B.
Table 1. Preliminary mean lifetimes and reduced electromagnetic transition probabilities in the yrast band of ${ }^{124} \mathrm{Ba}$ derived in this work. The values shown in the table are the average from the results for forward and backward rings.

\begin{tabular}{lllll}
\hline $\begin{array}{l}\mathrm{E}_{l e v} \\
{[\mathrm{keV}]}\end{array}$ & $\mathrm{I}^{\pi}$ & $\begin{array}{l}\mathrm{E}_{\gamma} \\
{[\mathrm{keV}]}\end{array}$ & $\begin{array}{l}\tau \\
{[\mathrm{ps}]}\end{array}$ & $\begin{array}{l}B(E 2) \\
{\left[\mathrm{e}^{2} \mathrm{~b}^{2}\right]}\end{array}$ \\
\hline 651.7 & $4^{+}$ & 421.1 & $9.1(8)$ & $0.664(58)$ \\
1228.4 & $6^{+}$ & 576.5 & $2.1(2)$ & $0.6(1)$ \\
1923.3 & $8^{+}$ & 694.7 & $1.2(4)$ & $0.425(148)$ \\
\hline
\end{tabular}

The data was analyzed by using the computer program NAPATAU [15] developed at the Institut für Kernphysik at University of Cologne. The derived values of $\tau$ (the $\tau$ -curve) should not depend on the distance at which they have been determined and correspondingly should be constant when plotted versus distance. A deviation from such behavior immediately indicates the presence of systematic errors in the analysis. For each level the lifetime was extracted for both forward and backward angles and only the points in the region of sensitivity were used.

The state $4_{1}^{+}$has energy of $651.7 \mathrm{keV}$, and decays by a $421.1 \mathrm{keV}$ transition to the $2_{1}^{+}$state. Gating on the $6_{1}^{+} \rightarrow 4_{1}^{+}$ transition (which directly feeds this state) produces the $\gamma \gamma$ coincidence spectra shown as an example in Fig. 2. The preliminary mean value for the lifetime derived from these spectra via DDCM is $\tau\left(4_{1}^{+}\right)=9.1(8)$ ps. An example of DDCM analysis for the $4_{1}^{+}$state for backward angles using the program NAPATAU is shown in Fig. 3.

The $6_{1}^{+} \rightarrow 4_{1}^{+}$transition has a level energy of $576.5 \mathrm{keV}$. Gates were applied on the shifted component of the populating $8_{1}^{+} \rightarrow 6_{1}^{+}$transition at $694.7 \mathrm{keV}$ observed both under forward and backward angles. They resulted in spectra with low background and good statistics similar to those for the $421.1 \mathrm{keV}$ transition. The preliminary mean value for the lifetime for the $6_{1}^{+}$state is $\tau\left(6_{1}^{+}\right)=2.1(2) \mathrm{ps}$.

The lifetime of the $8_{1}^{+}$state was analyzed in the same way, by gating on the shifted component of the populating $10_{1}^{+} \rightarrow 8_{1}^{+}$transition at $764.4 \mathrm{keV}$. The preliminary lifetime for the $8_{1}^{+}$state is $\tau\left(8_{1}^{+}\right)=1.2(4) \mathrm{ps}$, without Doppler Shift Attenuation (DSA) corrections.

The current analysis of the RDDS data yields 3 preliminary lifetimes in the yrast band of ${ }^{124} \mathrm{Ba}$, summarized in Table 1.

The comparison of the normalized $B(E 2)$ values (calculated from the deduced lifetimes) with the theoretical predictions of the vibrator, rotor and $\mathrm{X}(5)$ models, are shown in Fig. 4. The figure shows that for $4_{1}^{+}$there is a good agreement with the predicted by $\mathrm{X}(5)$ value. However, the $B(E 2)$ value of the $6_{1}^{+} \rightarrow 4_{1}^{+}$transition deviates from $\mathrm{X}(5)$ and is getting closer to that expected for a rotor nucleus. At spin $8_{1}^{+}$, there is even bigger deviation that may be due to deep structural change as the nucleus enters the backbending region.

\section{Summary}

In the present work RDDS lifetime measurements were carried out. For the data analysis, the Differential Decay 


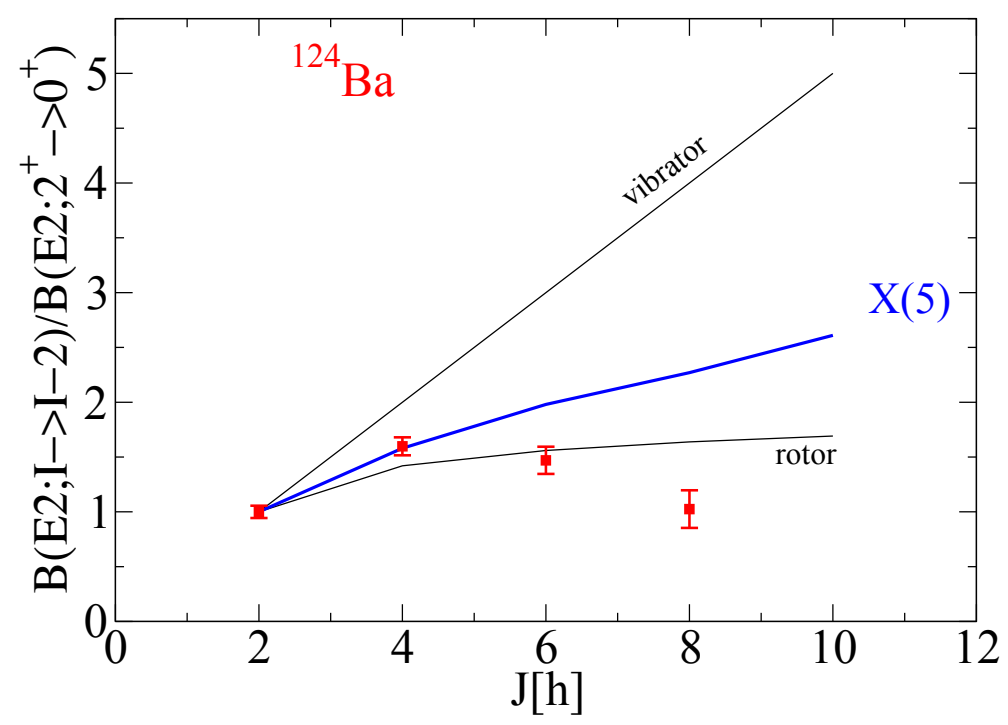

Figure 4. (Color online) Relative $B(E 2)$ (normalized to known from previous experiment $B\left(E 2 ; 2_{1}^{+} \rightarrow 0_{1}^{+}\right)[16]$ ) values measured in the g.s. band of ${ }^{124} \mathrm{Ba}$ compared to the vibrator, rotor and $\mathrm{X}(5)$ models prediction.

Curve method was employed. The lifetimes of several states in the g.s. band of ${ }^{124} \mathrm{Ba}$ were determined. The trend of the experimentally deduced normalized $B(E 2)$ strengths reveals a drop at the $8_{1}^{+}$state. This might be an indication for structural changes such as crossing with a two-quasiparticle configuration with different deformation. The DSA effects will be taken into account for determination of the final lifetimes.

This work is supported by the BgNSF grant DN08/23/2016 and by the partnership agreement between the University of Cologne and University of Sofia.

\section{References}

[1] K. Schiffer et al., Z. Phys. A 327, 251 (1987)

[2] K. Schiffer et al., Z. Phys. A 458, 337 (1986)

[3] Xiangfu Sun et al., Nucl. Phys. A 436, 506 (1985)

[4] T. Komatsubara et al., Nucl. Phys. A 496, 605 (1989)

[5] S. Pilotte et al., Nucl. Phys. A 514, 545 (1990)
[6] A. Al-Khatib et al., Phys. Rev. C 74, 014305 (2006)

[7] J. P. Martin et al., Nucl. Phys. A 489, 169 (1988)

[8] R. Wyss et al., Z. Phys. A 332, 241 (1989)

[9] F. Iachello, Phys. Rev. Lett. 87, 052502 (2001)

[10] A. Dewald et al., Prog. Part. Nucl. Phys. 67, 786 (2012)

[11] T. K. Alexander, J. S. Forster, Advances in Nuclear Physics (Plenum Press, New York, 1978) 197.

[12] P. Petkov, Nucl. Instrum. Methods Phys. Res. A 349, 289 (1994)

[13] A. Dewald, S. Harissopulos, P. Brentano, Z. Phys. A 334, 163 (1989)

[14] G. Böhm, A. Dewald, P. Petkov, and P. Brentano, Nucl. Instrum. Methods Phys. Res. A 329, 248 (1993)

[15] Program NAPATAU, University of Cologne (unpublished)

[16] K. Uchiyama, Eur. Phys. J A 2, 13-15 (1998) 